\title{
The FIB/SEM Technique, Atomic Force Microscopy and Acoustic Microscopy for Detection of Subsurface Defects in Thin DLC Coatings
}

\author{
P. V. Zinin*, M. H. Manghnani*, S. Berezina*, B. Koehler**, K. Bernland**, D. Fei***, and \\ D. A Rebinsky*** \\ * School of Ocean and Earth Science and Technology, University of Hawaii, Honolulu, USA \\ ** Fraunhofer IZFP-D, 01326 Dresden, Germany \\ *** Technical Center, Caterpillar Inc., Peoria, Illinois 61656, USA
}

The use of diamond-like carbon (DLC) coatings is becoming widely spread nowadays because the coating offers a significant improvement to wear and/or abrasion resistances of coated components without affecting their bulk properties. One of the key parameters that have great impact on coating's tribological properties is its microstructure; particularly, defect density, size and location. Defects can adversely affect the performance of the coating, leading to unpredicted premature failure of the coated component. In this work high frequency scanning acoustic microscopy (SAM) and focused ion beam/scanning electron microscopy (FIB/SEM) techniques were employed to characterize subsurface defects in chromium containing DLC (Cr-DLC) coatings.

A high frequency scanning acoustic microscope operating at 1-1.3 GHz was used to investigate subsurface defects in Cr-DLC coatings that were 2-3 $\mu \mathrm{m}$ thick. These Cr-DLC coatings were produced in Caterpillar Inc. in-house closed-field unbalanced magnetron sputtering system (CFUMS). Because the wavelength of the longitudinal wave in the coating was comparable to the coating thickness, the acoustical images obtained were near-field images. To interpret the features in the acoustical images, a multidisciplinary approach was utilized through the combination of SAM with atomic force microscopy (AFM), FIB technique, and optical microscopy.

Figures $1 \mathrm{a}$ and $1 \mathrm{~b}$ show the $1.3-\mathrm{GHz}$ acoustic images of a Cr-DLC coated specimen at a defocus of 0 $\mu \mathrm{m}$ and $-4 \mu \mathrm{m}$, respectively. Defects inside circles can be traced from the surface of the coating. Defects inside elipses appear only in the image at $z=-4 \mu \mathrm{m}$ and are subsurface defects.

To locate the depth and origin of the defects, the specimen was examined by using FIB/SEM technique. We traced a defect from the specimen surface to its disappearance. The specimen was positioned in a FIB/SEM imaging system. An ion sputtering process was applied from above the specimen to do fine milling of the coating. The specimen was then imaged with high resolution by SEM (Figure 2). With repeated sputtering and imaging, the 3-D microstructure of the coating was examined without removing or repositioning the specimen. The images obtained with the FIB technique showed that defects visible by SAM are located mainly at the interface between the DLC coating and steel substrate (at a depth of 3 $\mu \mathrm{m})$. It was found that while being sputtered away subsurface defects do not follow the same pattern as surface defects, which suggested that the underlying defect has different sputtering properties than the surrounding material.

A model for bump-like defects is proposed based on the SAM, EDX and FIB analysis results. The coatings have delaminations/voids between the $\mathrm{Cr}$ bond layer and steel substrate. These defects cause bump-like surface defects (Figs. 3a and b) that have a larger diameter than subsurface defects. 

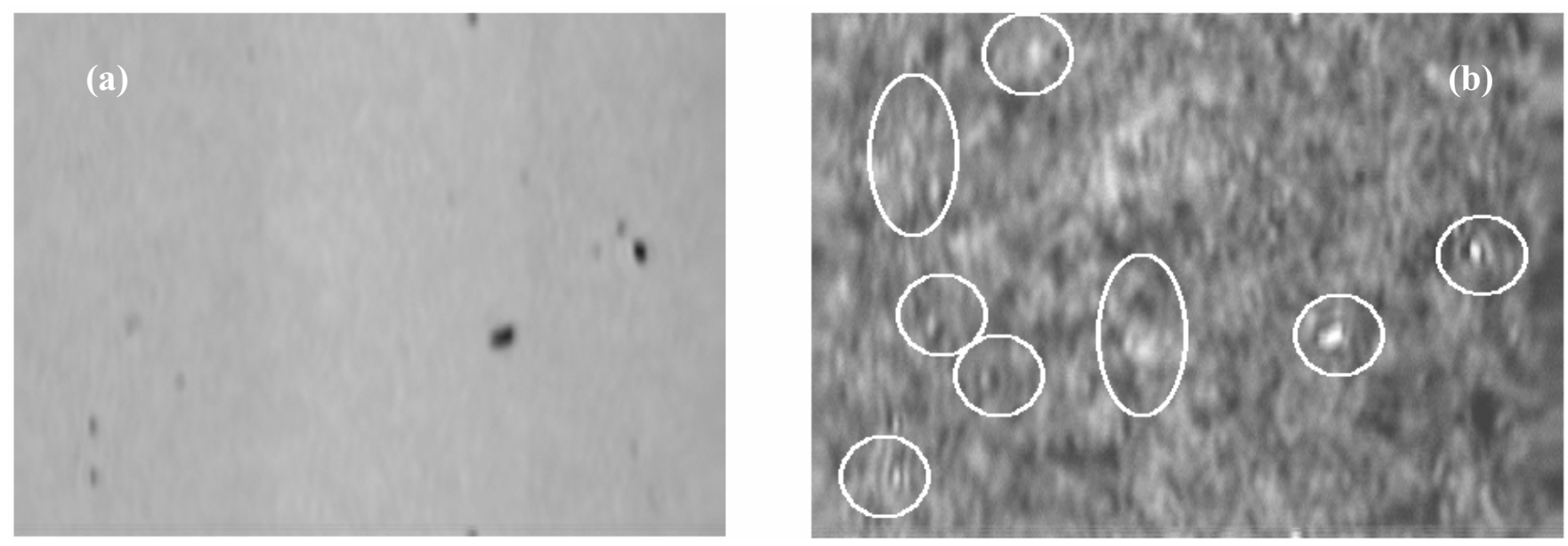

Fig.1. Acoustic image of specimen Cr-DLC \#624 at $1.3 \mathrm{GHz}$ where the horizontal image size was 100 $\mu \mathrm{m}$ with (a) defocus $\mathrm{z}=0 \mu \mathrm{m}$ and (b) defocus $\mathrm{z}=-4 \mu \mathrm{m}$.
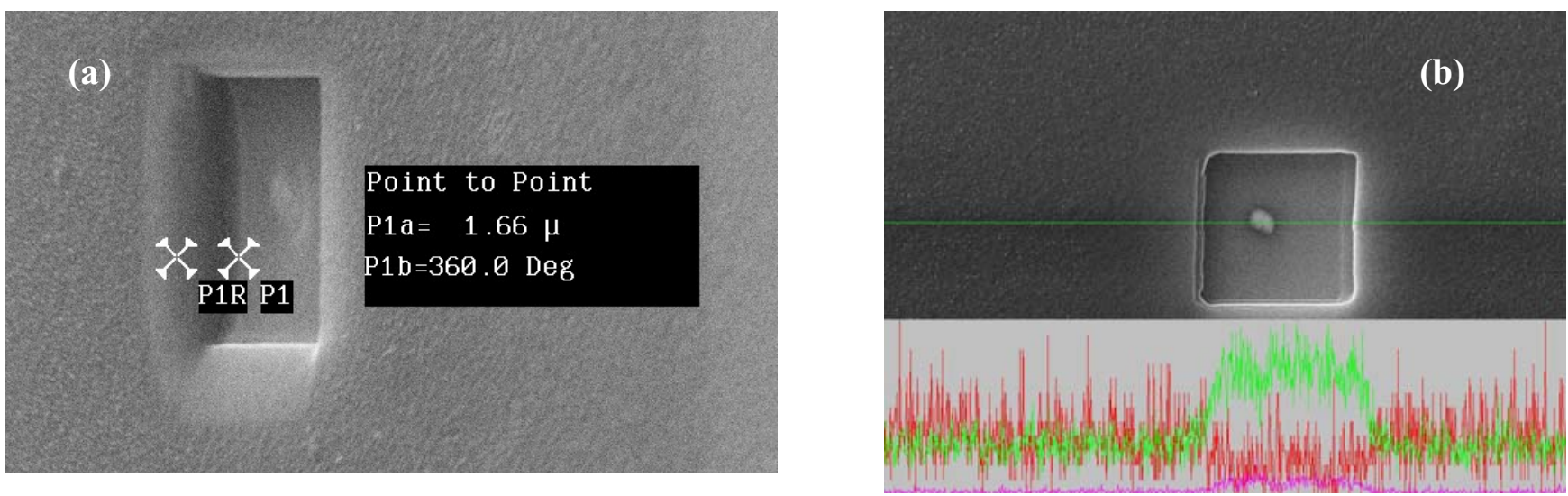

Fig. 2. SEM images of the Cr-DLC coating. (a) defect after 106 minutes of sputtering. The depth of the trench is approximately $1.9 \mu \mathrm{m}$. P1a is the distance between markets. (b) EDX-analysis results of the sputtered area, with no tilt of the image. Green $=$ chrome, purple $=$ iron, red $=$ carbon.
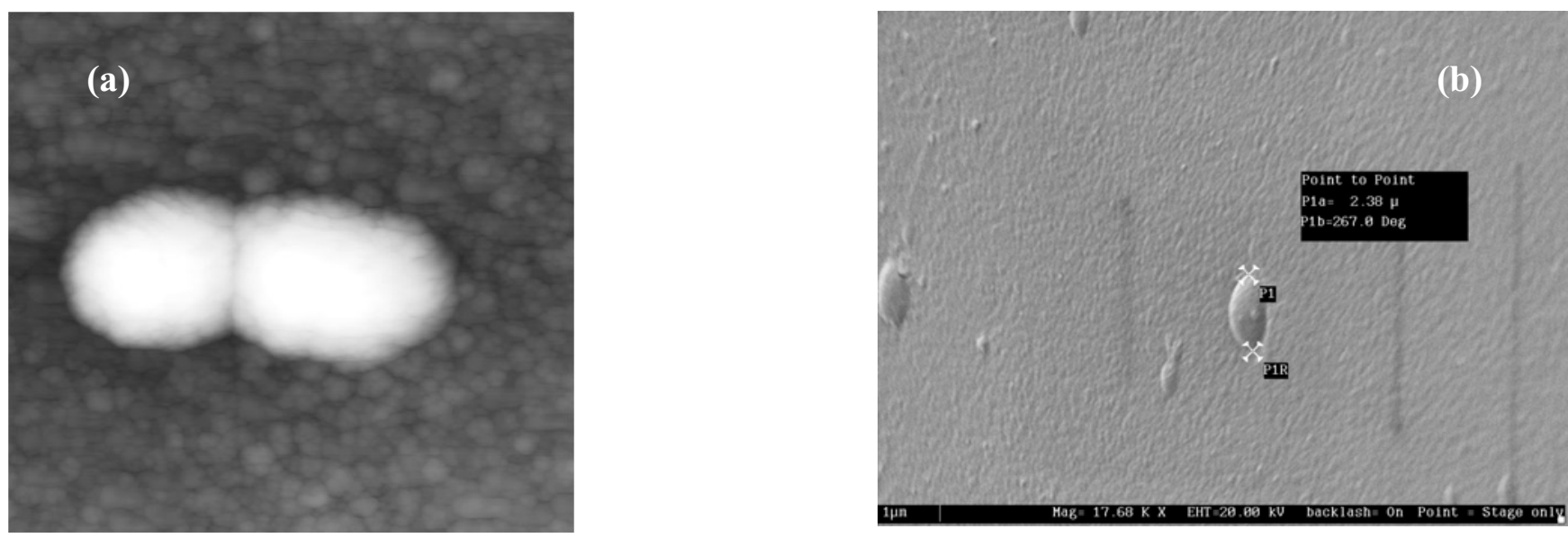

Fig. 3. Images of the Cr-DLC specimen (a) AFM image, deflection mode contrast with a field of view of $6 \times 6 \mu \mathrm{m}^{2}$; (b) SEM image showing the defect prior to sputtering. 